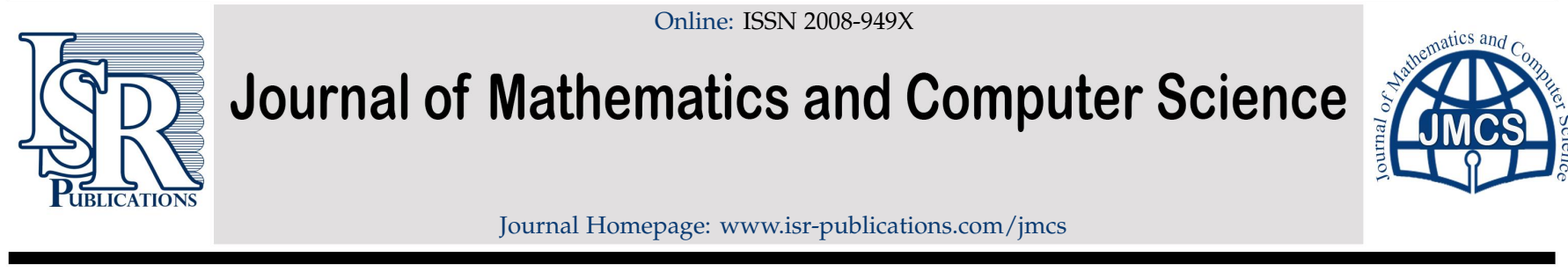

\title{
Empirical study of new iterative algorithm for generalized nonexpansive operators
}

\author{
Nisha Sharma ${ }^{\mathrm{a}}$, Lakshmi Narayan Mishra ${ }^{\mathrm{b}}$, Suyash Narayan Mishrac ${ }^{\mathrm{c}}$, Vishnu Narayan Mishra ${ }^{\mathrm{d}, *}$ \\ ${ }^{a}$ Department of Mathematics, Pt. J.L.N. Govt. College, Faridabad 121002, India. \\ ${ }^{b}$ Department of Mathematics, School of Advanced Sciences, Vellore Institute of Tech-nology (VIT) University, Vellore 632 014, Tamil \\ Nadu, India. \\ ${ }^{c}$ Department of Applied Sciences, Institute of Engineering and Technology (IET), Lucknow, 226 021, Uttar Pradesh, India. \\ ${ }^{d}$ Department of Mathematics, Faculty of Science, Indira Gandhi National Tribal University, Lalpur, Amarkantak, Anuppur 484 887, \\ Madhya Pradesh, India.
}

\begin{abstract}
The focal objective of this paper is to establish a new iterative algorithm, namely $\nu_{n}$ and utilize the same to prove some strong and weak convergence results in Banach spaces. An example is given to confirm the efficiency of aforementioned scheme. Since the iteration $\mathcal{V}_{n}$ is faster than many existing iterative algorithms, so the results in this paper are the extensions, improvements and the generalizations in the existing literature of fixed point in Banach spaces.
\end{abstract}

Keywords: Fixed point, strong convergence, weak convergence, generalized nonexpansive mapping, Banach space.

2020 MSC: 47H09, 47H10.

(C)2022 All rights reserved.

\section{Introduction}

Throughout this paper, we will denote set of natural numbers by $\mathbb{N}$ and set of real numbers by $\mathbb{R}$. A mapping $\wp$ on a subset $\mathcal{B}_{\mathrm{s}}$ of a Banach space $\mathcal{B}$ is said to be nonexpansive if

$$
\|\wp x-\wp y\| \leqslant\|x-y\|, \text { for all } x, y \in \mathcal{B}_{s} .
$$

An element $\mathrm{q} \in \mathcal{B}_{\mathrm{s}}$ is said to be a fixed point of $\wp$ if $\mathrm{q}=\wp \mathrm{q}$. From now on, we will denote set of all fixed points of $\wp$ by $\mathfrak{f}_{\wp}$. A mapping $\wp: \mathcal{B}_{s} \rightarrow \mathcal{B}_{s}$ is said to be quasi-nonexpansive mapping, if $\mathfrak{f}_{\wp} \neq \emptyset$ and $\|\wp x-\sigma\| \leqslant\|x-\sigma\|$ for all $x \in \mathcal{B}_{s}$ and $\sigma \in \mathfrak{f}_{\wp}$. The existence of fixed points for nonexpansive mappings in the setting of Banach spaces was studied independently by Browder [4], Gohde [5], and Kirk [7]. They proved that if $\mathcal{B}_{s}$ is nonempty closed bounded and convex subset of a uniformly convex Banach space, then every nonexpansive mapping $\wp: \mathcal{B}_{\mathrm{s}} \rightarrow \mathcal{B}_{\mathrm{s}}$ has atleast one fixed point. A number of generalizations of nonexpansive mappings have been studied by numerous authors in recent years.

\footnotetext{
${ }^{*}$ Corresponding author

Email address: vishnunarayanmishra@gnail.com, vnm@igntu.ac.in (Vishnu Narayan Mishra)

doi: $10.22436 /$ jmcs.025.03.07
}

Received: 2020-12-25 Revised: 2021-03-15 Accepted: 2021-05-21 
In [16] Suzuki introduced a new class of mappings (weaker than nonexpansiveness and stronger than quasi-nonexpansiveness) known as Suzuki generalized nonexpansive mappings which is a condition on mappings called condition $(\mathcal{C})$ and obtained successfully some convergence and existence results for such mappings. A mapping $\wp: \mathcal{B}_{s} \rightarrow \mathcal{B}_{s}$ is said to satisfy condition (C) (sometimes Suzuki generalized nonexpansive) if

$$
\frac{1}{2}\|x-\wp x\| \leqslant \sigma\|x-y\| \text { implies }\|\wp x-\wp y\| \leqslant\|x-y\|,
$$

for each $x, y \in \mathcal{B}_{s}$. Suzuki proved that condition $(\mathcal{C})$ is weaker than nonexpansiveness and stronger than quasi nonexpansiveness.

It is natural to study the computation of fixed points for the known existence results, which is not an easy task. The Banach contraction mapping principle uses Picard iteration process $x_{n+1}=\wp x_{n}$ for approximation of the unique fixed point. Some other well-known iteration schemes are Mann [8], Ishikawa [6], Agarwal [3], Noor [10], Abbas [1], Thakur et al. [18], Mishra et al. [9] and so on. Speed of convergence plays an important role for an iteration process to be preferred on another iteration process. Rhoades [12] mentioned that the Mann iteration process for decreasing function converge faster than the Ishikawa iteration process and for increasing function the Ishikawa iteration process is better than the Mann iteration process.

The well-known Mann [8] and Ishikawa [6] iteration processes are respectively defined as:

$$
\left\{\begin{array}{l}
x_{1} \in \mathcal{B}_{s} \\
x_{n+1}=\left(1-\eta_{n}^{0}\right) x_{n}+\eta_{n}^{0} \wp x_{n}, n \in \mathbb{N}
\end{array}\right.
$$

where $\eta_{n}^{0} \in(0,1)$ and

$$
\left\{\begin{array}{l}
x_{1} \in \mathcal{B}_{s}, \\
y_{n}=\left(1-\eta_{n}^{1}\right) x_{n}+\eta_{n}^{1} \wp x_{n}, \\
x_{n+1}=\left(1-\eta_{n}^{0}\right) x_{n}+\eta_{n}^{0} \wp y_{n}, n \in \mathbb{N}
\end{array}\right.
$$

where $\eta_{n}^{0}, \eta_{n}^{1} \in(0,1)$.

In 2007, Agarwal et al. [3] introduced the following iteration process known as S iteration:

$$
\left\{\begin{array}{l}
x_{1} \in \mathcal{B}_{s} \\
y_{n}=\left(1-\eta_{n}^{1}\right) x_{n}+\eta_{n}^{1} \wp x_{n} \\
x_{n+1}=\left(1-\eta_{n}^{0}\right) \wp x_{n}+\eta_{n}^{0} \wp y_{n}, n \in \mathbb{N}
\end{array}\right.
$$

where $\eta_{n}^{0}, \eta_{n}^{1} \in(0,1)$. They proved that the rate of convergence of iteration process of (1.1) is same to the picard iteration and faster than Mann iteration process in the class of contraction mappings.

Thakur et al. [18] used a new iteration process, defined as:

$$
\left\{\begin{array}{l}
x_{1} \in \mathcal{B}_{s} \\
z_{n}=\left(1-\eta_{n}^{1}\right) x_{n}+\eta_{n}^{1} \wp x_{n} \\
y_{n}=\wp\left(\left(1-\eta_{n}^{0}\right) x_{n}+\eta_{n}^{0} z_{n}\right) \\
x_{n+1}=\wp y_{n}, n \in \mathbb{N}
\end{array}\right.
$$

where $\eta_{\mathfrak{n}}^{0}, \eta_{\mathfrak{n}}^{1} \in(0,1)$. With the help of numerical example, they proved that (1.2) is faster than Picard, Mann, Ishikawa, Agarwal, Noor and Abbas iteration process in the class of Suzuki generalized nonexpansive mappings.

Recently in 2018, Ullah and Arshad [19] introduced $\mathrm{K}^{*}$ iteration process:

$$
\left\{\begin{array}{l}
x_{1} \in \mathcal{B}_{s} \\
z_{n}=\left(1-\eta_{n}^{1}\right) x_{n}+\eta_{n}^{1} \wp x_{n} \\
y_{n}=\wp\left(\left(1-\eta_{n}^{0}\right) z_{n}+\eta_{n}^{0} \wp z_{n}\right) \\
x_{n+1}=\wp y_{n}, n \in \mathbb{N}
\end{array}\right.
$$


where $\eta_{n}^{0}, \eta_{n}^{1} \in(0,1)$. They claimed that the rate of convergence of iteration (1.4) is faster than all above mentioned iteration schemes. Now, inspired by the works mentioned above, we propose the following problem:

Question 1.1. Is it possible to develop an iteration process whose rate of convergence is even faster than the iteration process (1.3)?

As an answer, we introduce the following new iteration called as $\mathcal{V}_{n}$ iteration process:

$$
\left\{\begin{array}{l}
x_{1} \in \mathcal{B}_{s} \\
z_{n}=\wp x_{n} \\
y_{n}=\wp\left(\left(1-\eta_{n}^{1}\right) \wp z_{n}+\eta_{n}^{1} z_{n}\right), \\
x_{n+1}=\wp\left(\left(1-\eta_{n}^{0}\right) y_{n}+\eta_{n}^{0} \wp y_{n}\right), n \in \mathbb{N}
\end{array}\right.
$$

where $\eta_{n}^{0}, \eta_{n}^{1} \in(0,1)$. In this way, we approximate fixed points of Suzuki generalized nonexpansive mappings using (1.4). With the help of numerical example, we compare the rate of convergence of our new $\mathcal{V}_{\mathrm{n}}$ iteration scheme with the existing faster iteration schemes.

\section{Preliminaries}

In this section, we give some preliminaries. Let $\mathcal{B}$ be a Banach space and $\mathcal{B}_{\mathrm{s}}$ be a nonempty closed convex subset of $\mathcal{B}$. Let $\left\{x_{n}\right\}$ be a bounded sequence in $\mathcal{B}_{s}$. For $x \in \mathcal{B}_{s}$, set

$$
r\left(x,\left\{x_{n}\right\}\right)=\limsup _{n \rightarrow \infty}\left\|x-x_{n}\right\| .
$$

The asymptotic radius of $\left\{x_{n}\right\}$ relative to $\mathcal{B}_{s}$ is given by

$$
r\left(\mathcal{B}_{s},\left\{x_{n}\right\}\right)=\inf \left\{r\left(x,\left\{x_{n}\right\}\right): x \in \mathcal{B}_{s}\right\} .
$$

The asymptotic center of $\left\{x_{n}\right\}$ relative to $\mathcal{B}_{s}$ is the set

$$
A\left(\mathcal{B}_{s},\left\{x_{n}\right\}\right)=\left\{x \in \mathcal{B}_{s}: r\left(x,\left\{x_{n}\right\}\right)=r\left(\mathcal{B}_{s},\left\{x_{n}\right\}\right)\right\} .
$$

It is well-known that in a uniformly convex Banach spaces, $\mathrm{A}\left(\mathcal{B}_{s}, x_{n}\right)$ consists of exactly one point. Also, $A\left(\mathcal{B}_{s}, x_{n}\right)$ is nonempty and convex in the case when $\mathcal{B}_{s}$ is weakly compact and convex (see $\left.[2,17]\right)$.

Following are some basic definitions and results.

Definition 2.1. A Banach space $\mathcal{B}$ is said to be uniformly convex if for each $\varepsilon \in(0,2]$, there is a $\lambda>0$ such that for every $x, y \in \mathcal{B},\|x\| \leqslant 1,\|y\| \leqslant 1$, and $\|x-y\|>\varepsilon$, we have

$$
\frac{1}{2}\|x+y\| \leqslant(1-\lambda)
$$

Definition 2.2 ([11]). A Banach space $\mathcal{B}$ is said to have Opial's property if for each sequence $\left\{x_{n}\right\}$ in $\mathcal{B}_{\mathrm{s}}$ which weakly converges to $x \in \mathcal{B}$ and for every $y \in \mathcal{B}_{s}$, it follows the following

$$
\limsup _{n \rightarrow \infty}\left\|x_{n}-x\right\|<\limsup _{n \rightarrow \infty}\left\|x_{n}-y\right\|
$$

Examples of Banach spaces satisfying this condition are Hilbert spaces and all $l^{p}$ spaces $(1<p<\infty)$.

We now list some basic facts about Suzuki generalized nonexpansive mappings, which can be found in [16]. 
Proposition 2.3. Let $\mathcal{B}$ be a Banach space, $\mathcal{B}_{\mathrm{s}}$ a nonempty subset of $\mathcal{B}$, and $\wp: \mathcal{B}_{\mathrm{s}} \rightarrow \mathcal{B}_{\mathrm{s}}$ be a mapping.

(i) If $\wp$ is nonexpansive mapping then $\wp$ is a Suzuki generalized nonexpansive mapping.

(ii) If $\wp$ is Suzuki generalized nonexpansive mapping and has a fixed point, then $\wp$ is a quasi-nonexpansive mapping.

(iii) If $\wp$ is Suzuki generalized nonexpansive mapping, then $\mathfrak{f}_{\wp}$ is closed. Moreover, if $\mathcal{B}_{\mathrm{s}}$ is strictly convex and $\mathcal{B}_{\mathrm{s}}$ is convex, then $\mathfrak{f}_{\wp}$ is also convex.

(iv) If $\wp$ is Suzuki generalized nonexpansive mapping, then for each $x, y \in \mathcal{B}_{s}$,

$$
\|x-\wp(y)\| \leqslant 3\|x-\wp(x)\|+\|x-y\| .
$$

(v) If $\mathcal{B}_{\mathrm{s}}$ has Opial property, $\wp$ is Suzuki generalized nonexpansive, $\left\{x_{n}\right\}$ converges weakly to a point $w$, and $\lim _{n \rightarrow \infty}\left\|\wp x_{n}-x_{n}\right\|=0$, then $w \in \mathfrak{f}_{\wp}$.

Lemma 2.4 ([16]). Let $\mathcal{B}_{\mathrm{s}}$ be a weakly compact convex subset of a uniformly convex Banach space $\mathcal{B}$. Let $\wp$ be a mapping on $\mathcal{B}_{\mathrm{s}}$. Assume that $\wp$ is Suzuki generalized nonexpansive mapping. Then $\wp$ has a fixed point.

The following useful Lemma can be found in [13].

Lemma 2.5. Let $\mathcal{B}_{\mathrm{s}}$ be a uniformly convex Banach space and $0<\mathrm{p} \leqslant \eta_{\mathrm{n}}^{0} \leqslant \mathrm{q}<1$ for every $\mathrm{n} \in \mathbb{N}$. If $\left\{\mathrm{t}_{\mathrm{n}}\right\}$ and $\left\{s_{n}\right\}$ are two sequences in $\mathcal{B}_{s}$ such that $\limsup _{n \rightarrow \infty}\left\|\wp_{n}\right\| \leqslant \mathcal{B}_{s}, \limsup _{n \rightarrow \infty}\left\|s_{n}\right\| \leqslant c$, and $\lim _{n \rightarrow \infty} \| \eta_{n}^{0} t_{n}+$ $\left(1-\eta_{n}^{0}\right) s_{n} \|=c$ for some $c \geqslant 0$, then, $\lim _{n \rightarrow \infty}\left\|\wp_{n}-s_{n}\right\|=0$. Also, let $\wp$ be a Suzuki generalized nonexpansive mapping defined on a subset $\mathcal{B}_{\mathrm{s}}$ of a Banach space $\mathcal{B}$ with the Opial property. If a sequence $\left\{x_{n}\right\}$ converges weakly to $z$ and $\lim _{n \rightarrow \infty}\left\|\wp x_{n}-x_{n}\right\|=0$, then $I-\wp$ is demiclosed at zero.

\section{Convergence theorems in uniformly Banach spaces}

We start this section with following important Lemma.

Lemma 3.1. Let $\mathcal{B}_{\mathrm{s}}$ be a nonempty closed convex subset of a Banach space $\mathcal{B}$ and $\wp: \mathcal{B}_{\mathrm{s}} \rightarrow \mathcal{B}_{\mathrm{s}}$ be a Suzuki generalized nonexpansive mapping with $\mathfrak{f}_{\wp} \neq \emptyset$. Let $\left\{x_{n}\right\}$ be a sequence generated by (1.4), then $\lim _{n \rightarrow \infty}\left\|x_{n}-\sigma\right\|$ exists for each $\mathrm{q} \in \mathfrak{f}_{\wp}$.

Proof. Let $\mathrm{q} \in \mathfrak{f}_{\wp}$. By Proposition 2.3 part (ii), we have

$$
\begin{aligned}
\left\|y_{n}-\sigma\right\| & =\left\|\wp\left(\left(1-\eta_{n}^{1}\right) \wp z_{n}+\eta_{n}^{1} z_{n}\right)-\sigma\right\| \\
& \leqslant\left\|\left(1-\eta_{n}^{1}\right) \wp z_{n}+\eta_{n}^{1} z_{n}-\sigma\right\| \\
& \leqslant\left(1-\eta_{n}^{1}\right)\left\|\wp z_{n}-\sigma\right\|+\eta_{n}^{1}\left\|z_{n}-\sigma\right\| \\
& \leqslant\left(1-\eta_{n}^{1}\right)\left\|z_{n}-\sigma\right\|+\eta_{n}^{1}\left\|z_{n}-\sigma\right\| \\
& =\left\|z_{n}-\sigma\right\| \\
& =\left\|\wp x_{n}-\sigma\right\| \\
& \leqslant\left\|x_{n}-\sigma\right\|,
\end{aligned}
$$

which implies

$$
\begin{aligned}
\left\|x_{n+1}-\sigma\right\| & =\left\|\wp\left(\left(1-\eta_{n}^{0}\right) y_{n}+\eta^{0} \wp y_{n}\right)-\sigma\right\| \\
& \leqslant\left\|\left(1-\eta_{n}^{0}\right) y_{n}+\eta_{n}^{0} \wp y_{n}-\sigma\right\| \\
& \leqslant\left(1-\eta_{n}^{0}\right)\left\|y_{n}-\sigma\right\|+\eta_{n}^{0}\left\|\wp y_{n}-\sigma\right\| \\
& \leqslant\left(1-\eta_{n}^{0}\right)\left\|y_{n}-\sigma\right\|+\eta_{n}^{0}\left\|y_{n}-\sigma\right\| \\
& =\left\|y_{n}-\sigma\right\| \\
& \leqslant\left\|x_{n}-\sigma\right\| .
\end{aligned}
$$

Thus $\left\{\left\|x_{n}-\sigma\right\|\right\}$ is bounded and non increasing, which implies that $\lim _{n \rightarrow \infty}\left\|x_{n}-\sigma\right\|$ exists for each $\mathrm{q} \in \mathfrak{f}_{\wp}$. 
The following theorem is useful for the next results.

Theorem 3.2. Let $\mathcal{B}_{\mathrm{s}}$ be a nonempty closed convex subset of a uniformly convex Banach space $\mathcal{B}$ and $\wp: \mathcal{B}_{\mathrm{s}} \rightarrow \mathcal{B}_{\mathrm{s}}$ a Suzuki generalized nonexpansive mapping. Let $\left\{x_{n}\right\}$ be a sequence generated by $(A)$. Then, $\mathfrak{f}_{\wp} \neq \emptyset$ if and only if $\left\{x_{n}\right\}$ is bounded and $\lim _{n \rightarrow \infty}\left\|\wp x_{n}-x_{n}\right\|=0$.

Proof. Suppose that $\mathfrak{f}_{\wp} \neq \emptyset$ and $q \in \mathfrak{f}_{\wp}$. Then, by Lemma 3.1, $\lim _{n \rightarrow \infty}\left\|x_{n}-\sigma\right\|$ exists and $\left\{x_{n}\right\}$ is bounded. Put

$$
\lim _{n \rightarrow \infty}\left\|x_{n}-\sigma\right\|=c .
$$

By the proof of Lemma 3.1, $\left\|z_{n}-\sigma\right\| \leqslant \sigma\left\|x_{n}-\sigma\right\|$ and $\left\|y_{n}-\sigma\right\| \leqslant \sigma\left\|x_{n}-\sigma\right\|$. It follows that

$$
\limsup _{n \rightarrow \infty}\left\|z_{n}-\sigma\right\| \leqslant \limsup _{n \rightarrow \infty}\left\|x_{n}-\sigma\right\|=c,
$$

and

$$
\limsup _{n \rightarrow \infty}\left\|y_{n}-\sigma\right\| \leqslant \limsup _{n \rightarrow \infty}\left\|x_{n}-\sigma\right\|=c .
$$

By Proposition 2.3 part (ii), we have

$$
\limsup _{n \rightarrow \infty}\left\|\wp z_{n}-\sigma\right\| \leqslant \limsup _{n \rightarrow \infty}\left\|z_{n}-\sigma\right\| \leqslant \limsup _{n \rightarrow \infty}\left\|x_{n}-\sigma\right\|=c .
$$

Again by the proof of Lemma 3.1, $\left\|x_{n+1}-\sigma\right\| \leqslant \sigma\left\|y_{n}-\sigma\right\|$. It follows that

$$
c \leqslant \liminf _{n \rightarrow \infty}\left\|y_{n}-\sigma\right\|
$$

From (3.3) and (3.5), we obtain

$$
c=\lim _{n \rightarrow \infty}\left\|y_{n}-\sigma\right\|
$$

From (3.1) and (3.6), we have

$$
\begin{aligned}
c & =\lim _{n \rightarrow \infty}\left\|y_{n}-\sigma\right\| \\
& =\lim _{n \rightarrow \infty}\left\|\wp\left(\left(1-\eta_{n}^{1}\right) \wp z_{n}+\eta_{n}^{1} z_{n}\right)-\sigma\right\| \\
& \leqslant \lim _{n \rightarrow \infty}\left\|\left(1-\eta_{n}^{1}\right) \wp z_{n}+\eta_{n}^{1} z_{n}-\sigma\right\| \\
& =\lim _{n \rightarrow \infty}\left\|\left(1-\eta_{n}^{1}\right)\left(\wp z_{n}-q\right)+\eta_{n}^{1}\left(z_{n}-q\right)\right\| \\
& \leqslant \lim _{n \rightarrow \infty}\left(1-\eta_{n}^{1}\right)\left\|\wp z_{n}-\sigma\right\|+\lim _{n \rightarrow \infty} \eta_{n}^{1}\left\|z_{n}-\sigma\right\| \\
& \leqslant \lim _{n \rightarrow \infty}\left(1-\eta_{n}^{1}\right)\left\|z_{n}-\sigma\right\|+\lim _{n \rightarrow \infty} \eta_{n}^{1}\left\|z_{n}-\sigma\right\| \\
& =\lim _{n \rightarrow \infty}\left\|z_{n}-\sigma\right\| \\
& \leqslant \lim _{n \rightarrow \infty}\left\|x_{n}-\sigma\right\| \\
& =c .
\end{aligned}
$$

Hence,

$$
c=\lim _{n \rightarrow \infty}\left\|\left(1-\eta_{n}^{1}\right)\left(\wp z_{n}-q\right)+\eta_{n}^{1}\left(z_{n}-q\right)\right\| .
$$

Now, from (3.2), (3.4), and (3.6) together with Lemma 2.5, we obtain

$$
\lim _{n \rightarrow \infty}\left\|\wp z_{n}-z_{n}\right\|=0
$$

Now, it can easily be concluded that

$$
\lim _{n \rightarrow \infty}\left\|\wp x_{n}-x_{n}\right\|=\lim _{n \rightarrow \infty}\left\|\wp x_{n}-q+q-x_{n}\right\|=\lim _{n \rightarrow \infty}\left\|\wp x_{n}-\sigma\right\|+\left\|q-x_{n}\right\| \leqslant 2 \lim _{n \rightarrow \infty}\left\|x_{n}-\sigma\right\| .
$$


Since,

$$
\begin{aligned}
\lim _{n \rightarrow \infty}\left\|x_{n}-p\right\| & =\lim _{n \rightarrow \infty}\left\|\wp\left(\left(1-\eta_{n}^{0}\right) y_{n}+\eta_{n}^{0} \wp y_{n}\right)-\sigma\right\| \\
& =\lim _{n \rightarrow \infty}\left\|\left(1-\eta_{n}^{0}\right) y_{n}+\eta_{n}^{0} \wp y_{n}-\sigma\right\|=\lim _{n \rightarrow \infty}\left\|\left(1-\eta_{n}^{0}\right)\left(y_{n}-q\right)+\eta_{n}^{0}\left(\wp y_{n}-q\right)\right\|
\end{aligned}
$$

which on combining above equations and Lemma 2.5,

$$
\lim _{n \rightarrow \infty}\left\|y_{n}-\wp y_{n}\right\|=0
$$

and hence, using $\lim _{n \rightarrow \infty}\left\|y_{n}-p\right\|$, we have

$$
\lim _{n \rightarrow \infty}\left\|z_{n}-\wp z_{n}\right\|=0
$$

Also,

$$
\begin{aligned}
\left\|y_{n}-\wp z_{n}\right\| & =\left\|\wp\left(\left(1-\eta_{\mathfrak{n}}^{1}\right) \wp z_{n}+\eta_{\mathfrak{n}}^{1} z_{\mathfrak{n}}\right)-\wp z_{\mathfrak{n}}\right\| \\
& \leqslant\left\|\left(1-\eta_{\mathfrak{n}}^{1}\right) \wp z_{\mathfrak{n}}+\eta_{\mathfrak{n}}^{1} z_{\mathfrak{n}}-\wp z_{\mathfrak{n}}\right\| \\
& \leqslant\left\|\left(1-\eta_{\mathfrak{n}}^{1}\right) \wp z_{n}+\eta_{\mathfrak{n}}^{1} z_{\mathfrak{n}}-z_{\mathfrak{n}}\right\| \\
& \leqslant\left(1-\eta_{n}^{1}\right)\left\|\wp z_{\mathfrak{n}}-z_{\mathfrak{n}}\right\|,
\end{aligned}
$$

which implies that

$$
\lim _{n \rightarrow \infty}\left\|y_{n}-\wp y_{n}\right\|=0
$$

Also,

$$
\left\|z_{n}-y_{n}\right\| \leqslant\left\|z_{n}-\wp z_{n}\right\|+\left\|\wp z_{n}-y_{n}\right\| \Longrightarrow \lim _{n \rightarrow \infty}\left\|y_{n}-z_{n}\right\|=0
$$

now, equation (3.7) results

$$
\begin{aligned}
\lim _{n \rightarrow \infty}\left\|\wp x_{n}-x_{n}\right\| & \leqslant 2 \lim _{n \rightarrow \infty}\left\|y_{n}-\wp y_{n}\right\| \\
& \leqslant 2\left[\lim _{n \rightarrow \infty}\left\|y_{n}-\wp z_{n}+\wp z_{n}-\wp y_{n}\right\|\right] \\
& \leqslant 2\left[\lim _{n \rightarrow \infty}\left\|y_{n}-\wp z_{n}\right\|+\lim _{n \rightarrow \infty}\left\|\wp z_{n}-\wp y_{n}\right\|\right] \\
& \leqslant 2\left[\lim _{n \rightarrow \infty}\left\|y_{n}-\wp z_{n}\right\|+\lim _{n \rightarrow \infty}\left\|z_{n}-y_{n}\right\|\right]
\end{aligned}
$$

and hence, we have

$$
\lim _{n \rightarrow \infty}\left\|\wp x_{n}-x_{n}\right\|=0 \text {. }
$$

Conversely, we assume that $\left\{x_{n}\right\}$ is bounded and $\lim _{n \rightarrow \infty}\left\|\wp x_{n}-x_{n}\right\|=0$. Let $\left.q \in A \mathcal{B}_{s},\left\{x_{n}\right\}\right)$. By Proposition 2.3 part (iv), we have

$$
r\left(\wp \mathbf{q},\left\{x_{n}\right\}\right)=\limsup _{n \rightarrow \infty}\left\|x_{n}-\wp \sigma\right\| \leqslant 3 \limsup _{n \rightarrow \infty}\left\|x_{n}-\sigma\right\|+\limsup _{n \rightarrow \infty}\left\|x_{n}-\sigma\right\|=\limsup _{n \rightarrow \infty}\left\|x_{n}-\sigma\right\|=r\left(q,\left\{x_{n}\right\}\right) .
$$

Hence, we conclude that $\wp q \in A\left(\wp,\left\{x_{n}\right\}\right.$. Since $\mathcal{B}_{s}$ is uniformly convex, $A\left(\wp,\left\{x_{n}\right\}\right)$ consists of a unique element. Thus, we have $\wp q=q$. 
First we prove our weak convergence result.

Theorem 3.3. Let $\mathcal{B}_{\mathrm{s}}$ be a uniformly Banach space with Opial property, $\mathcal{B}_{\mathrm{s}}$ a nonempty closed convex subset of $\mathcal{B}$ and $\wp: \mathcal{B}_{\mathrm{s}} \rightarrow \mathcal{B}_{\mathrm{s}}$ be Suzuki generalized nonexpansive mapping with $\mathfrak{f}_{\wp} \neq \emptyset$. Then, $\left\{x_{n}\right\}$ generated by (1.4) converges weakly to an element of $\mathfrak{f}_{\wp}$.

Proof. By Theorem 3.2, $\left\{x_{n}\right\}$ is bounded and $\lim _{n \rightarrow \infty}\left\|\wp x_{n}-x_{n}\right\|=0$. Since $\mathcal{B}_{s}$ is uniformly convex, $\mathcal{B}_{s}$ is reflexive. So, subsequence $\left\{x_{n_{i}}\right\}$ of $\left\{x_{n}\right\}$ exists such that $\left\{x_{n_{i}}\right\}$ converges weakly to some $w_{1} \in \mathcal{B}_{s}$. By Proposition 2.3 part $(\mathrm{v})$, we have $w_{1} \in \mathfrak{f}_{\wp}$. It is sufficient to show that $\left\{x_{n}\right\}$ converges weakly to $w_{1}$. If not, then, there exists a subsequence $\left\{x_{n_{j}}\right\}$ of $\left\{x_{n}\right\}$ and $w_{2} \in \mathcal{B}_{s}$ such that $\left\{x_{n_{j}}\right\}$ converges weakly to $w_{2}$ and $w_{2} \neq w_{1}$. Again by Proposition 2.3 part $(\mathrm{v}), w_{2} \in \mathfrak{f}_{\wp}$. By Lemma 3.1 together with Opial property, we have

$$
\begin{aligned}
\lim _{n \rightarrow \infty}\left\|x_{n}-w_{1}\right\|=\lim _{i \rightarrow \infty}\left\|x_{n_{i}}-w_{1}\right\| & <\lim _{i \rightarrow \infty}\left\|x_{n_{i}}-w_{2}\right\| \\
& =\lim _{n \rightarrow \infty}\left\|x_{n}-w_{2}\right\| \\
& =\lim _{j \rightarrow \infty}\left\|x_{n_{j}}-w_{2}\right\|<\lim _{j \rightarrow \infty}\left\|x_{n_{j}}-w_{1}\right\|=\lim _{n \rightarrow \infty}\left\|x_{n}-w_{1}\right\| .
\end{aligned}
$$

This is a contradiction, so, $w_{1}=w_{2}$. Thus, $\left\{x_{n}\right\}$ converges weakly to $z_{1} \in \mathfrak{f}_{\wp}$.

Theorem 3.4. Let $\mathcal{B}_{\mathrm{s}}$ be a nonempty compact convex subset of a uniformly convex Banach space $\mathcal{B}$ and $\wp: \mathcal{B}_{\mathrm{s}} \rightarrow$ $\mathcal{B}_{s}$ be a Suzuki generalized nonexpansive mapping. Then, $\left\{x_{n}\right\}$ generated by (1.4) converges strongly to an element of $\mathfrak{f}_{\wp}$.

Proof. By Lemma $2.4, \mathfrak{f}_{\wp} \neq \emptyset$. By Theorem 3.2, $\lim _{n \rightarrow \infty}\left\|\wp x_{n}-x_{n}\right\|=0$. By compactness of $\mathcal{B}_{s}$, there exists a subsequence $\left\{x_{n_{j}}\right\}$ of $\left\{x_{n}\right\}$ such that $\left\{x_{n_{j}}\right\}$ converges strongly to $p$ for some $p \in \mathcal{B}_{s}$. By Proposition 2.3 (iv), we have

$$
\left\|x_{n_{j}}-\wp(p)\right\| \leqslant 3\left\|x_{n_{j}}-\wp\left(x_{n_{j}}\right)\right\|+\left\|x_{n_{j}}-p\right\| .
$$

Letting $j \rightarrow \infty$ we get $\wp p=p$. By Lemma 3.1, $\lim _{n \rightarrow \infty}\left\|x_{n}-p\right\|$ exists, for each $p \in \mathfrak{f}_{\wp}$. Therefore, we conclude that $\left\{x_{n}\right\}$ converges strongly to an element of $\mathfrak{f}_{\wp}$.

We now prove our strong convergence result.

Theorem 3.5. Let $\mathcal{B}_{\mathrm{s}}$ be a nonempty closed convex subset of a uniformly convex Banach space $\mathcal{B}$ and $\wp: \mathcal{B}_{\mathrm{s}} \rightarrow \mathcal{B}_{\mathrm{s}}$ be a Suzuki generalized nonexpansive mapping. If $\mathfrak{f}_{\wp} \neq \emptyset$ and $\lim \inf _{n \rightarrow \infty} \operatorname{dist}\left(x_{n}, \mathfrak{f}_{\wp}\right)=0\left(\right.$ where $\operatorname{dist}\left(x, \mathfrak{f}_{\wp}\right)=$ $\left.\inf \left\{\|x-\sigma\|: q \in \mathfrak{f}_{\wp}\right\}\right)$. Then, $\left\{x_{n}\right\}$ generated by (1.4) converges strongly to an element of $\mathfrak{f}_{\wp}$.

Proof. By Lemma 3.1, $\lim _{n \rightarrow \infty}\left\|x_{n}-\sigma\right\|$ exists, for each $q \in \mathfrak{f}_{\wp}$. So, $\lim _{n \rightarrow \infty} \operatorname{dist}\left(x_{n}, \mathfrak{f}_{\wp}\right)$ exists, thus

$$
\lim _{n \rightarrow \infty} \operatorname{dist}\left(x_{n}, \mathfrak{f}_{\wp}\right)=0 \text {. }
$$

Therefore, there exists subsequences $\left\{x_{n_{k}}\right\}$ of $\left\{x_{n}\right\}$ and $\left\{w_{n_{k}}\right\}$ in $\mathfrak{f}_{\wp}$ such that

$$
\left\|x_{n_{k}}-w_{n_{k}}\right\| \leqslant \frac{1}{2^{k}}
$$

for each $k \in \mathbb{N}$. By the proof of Lemma 3.1, $\left\{x_{n}\right\}$ is non increasing, so

$$
\left\|x_{n_{k+1}}-w_{k}\right\| \leqslant \sigma\left\|x_{n_{k}}-w_{n_{k}}\right\| \leqslant \frac{1}{2^{k}} .
$$

Therefore,

$$
\left\|w_{k+1}-w_{k}\right\| \leqslant\left\|w_{k+1}-x_{n_{k+1}}\right\|+\left\|x_{n_{k+1}}-w_{k}\right\| \leqslant \frac{1}{2^{k+1}}+\frac{1}{2^{k}} \leqslant \frac{1}{2^{k-1}} \rightarrow 0
$$


as $k \rightarrow \infty$. Hence, we conclude that $\left\{w_{k}\right\}$ is a Cauchy sequence in $\mathfrak{f}_{\wp}$ and so it converges to some q. Since, by Proposition 2.3 part (iii), $\mathfrak{f}_{\wp}$ is closed, then $q \in \mathfrak{f}_{\wp}$. By Lemma 3.1, $\lim _{n \rightarrow \infty}\left\|x_{n}-\sigma\right\|$ exists, hence $\left\{x_{n}\right\}$ converges strongly to $\mathrm{q} \in \mathfrak{f}_{\wp}$.

Definition 3.6 ([14]). A selfmap $\wp$ on $\mathcal{B}_{s}$ subset of a Banach space is said to satisfy condition (J) if there is a nondecreasing function $\psi: \mathbb{R}^{+} \rightarrow \mathbb{R}^{+}$with the property $\psi(0)=0$ and $\psi(s)>0$ for all $s \in(0, \infty)$ such that

$$
\|x-\wp x\| \leqslant \psi\left(\operatorname{dist}\left(x, \mathfrak{f}_{\wp}\right)\right)
$$

for all $x \in \mathcal{B}_{s}$, where

$$
\mathrm{d}\left(x, \mathfrak{f}_{\wp}\right)=\inf _{p \in \mathfrak{f}_{\wp}}\left\|x_{n}-p\right\| .
$$

Now, the strong convergence theorem using condition $(\mathcal{J})$ is as follows.

Theorem 3.7. Let $\mathcal{B}_{\mathrm{s}}$ be a nonempty closed convex subset of uniformly convex Banach space $\mathcal{B}$ and $\wp: \mathcal{B}_{\mathrm{s}} \rightarrow \mathcal{B}_{\mathrm{s}}$ be a Suzuki generalized nonexpansive mapping with $\mathfrak{f}_{\wp} \neq \emptyset$. If $\wp$ satisfies condition (J), then $\left\{x_{n}\right\}$ generated by (1.4) converges strongly to an element of $\mathfrak{f}_{\wp}$.

Proof. From Theorem 3.2, it follows that

$$
\liminf _{n \rightarrow \infty}\left\|\wp x_{n}-x_{n}\right\|=0
$$

Since $\wp$ satisfies condition $(\mathcal{J})$, we have

$$
\left\|x_{n}-\wp x_{n}\right\| \geqslant \psi\left(\operatorname{dist}\left(x_{n}, \mathfrak{f}_{\wp}\right)\right)
$$

From (3.8), we get

$$
\liminf _{n \rightarrow \infty}\left(\operatorname{dist}\left(x_{n}, \mathfrak{f}_{\infty}\right)\right)=0 .
$$

Since $\psi:[0, \infty) \rightarrow[0, \infty)$ is a nondecreasing function with $\psi(0)=0$ and $\psi(r)>0$ for all $r \in(0, \infty)$, we have

$$
\liminf _{n \rightarrow \infty} \operatorname{dist}\left(x_{n}, \mathfrak{f}_{\wp}\right)=0
$$

Therefore all the assumptions of Theorem 3.5 are satisfied and so $\left\{x_{n}\right\}$ converges strongly to an element of $\mathfrak{f}_{\wp}$.

\section{Numerical example}

In this section, an example is to be given which shows that $\mathcal{V}_{n}$ iteration scheme converges faster than the $\mathrm{K}^{*}$.

Example 4.1. Let $\mathcal{B}=(-\infty, \infty)$ and $\mathcal{B}_{s}=[1,50]$, and $\wp: \mathcal{B}_{s} \rightarrow \mathcal{B}_{s}$ be mapping defined as $\wp(x)=$ $\sqrt{x^{2}-8 x+48}$ for all $x \in \mathcal{B}_{s}$. Clearly, $x=6$ is the fixed point of $\wp$. Set $x_{0}=35$ for all $n \in \mathbb{N}, \eta_{n}^{0}=0.25$ and $\eta_{n}^{1}=0.65$. The iterative values for $x_{n}$ are given in Table 1. Graphical comparison can easily be shown by Figure 1. Clearly the new $\mathcal{V}_{\mathrm{n}}$ iteration process converging fast to the fixed point of $\wp$ as compared to other iteration processes whereas graphical comparison of $\mathcal{V}_{n}$ for the function $\frac{1+4 x}{5}$ for $\alpha_{n}=0.75$ and $\beta_{n}=0.65$ with initial value $x_{0}=10$ is shown by Fig. 2 .

Tabulated values for $\mathcal{V}_{n}, K^{*}$, and $S$ are respectively generated by (1.4), (1.3), and (1.1) iteration schemes for mapping $\wp$. 
Table 1: Empirical behavior of $\mathcal{V}_{\mathrm{n}}$ for initial value $x_{0}=35$.

\begin{tabular}{c|c|c|c|}
\hline Steps & $\mathbf{S}$ & $\boldsymbol{K}^{*}$ & $\boldsymbol{V}_{\boldsymbol{n}}$ \\
\hline 1 & 35 & 35 & 35 \\
2 & 30.955 & 25.0851 & 21.1992 \\
3 & 27.0005 & 16.0649 & 10.086 \\
4 & 23.1644 & 9.14354 & 6.17342 \\
5 & 19.4882 & 6.33968 & 6.0026 \\
6 & 16.0349 & 6.01932 & 6.00004 \\
7 & 12.9017 & 6.00102 & 6. \\
8 & 16.8399 & 6. & 6. \\
9 & 10.2332 & 6. & 6. \\
10 & 8.20932 & 6. & 6. \\
11 & 6.94566 & 6. & 6. \\
12 & 6.33739 & 6. & 6. \\
13 & 6.10764 & 6. & 6. \\
14 & 6.03275 & 6. & 6. \\
15 & 6.0098 & 6. & 6. \\
16 & 6.0029 & 6. & 6. \\
\hline
\end{tabular}

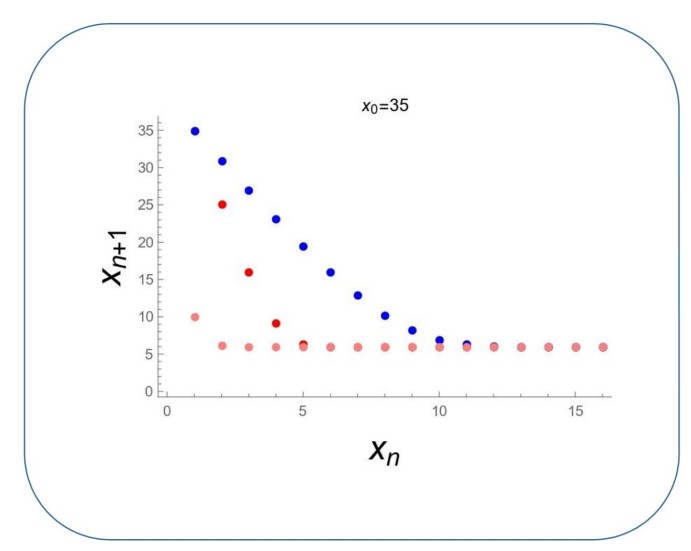

Figure 1: Graphical comparison to study the efficiency of $\mathcal{V}_{\mathrm{n}}$ as compared to $\mathrm{K}^{*}$ and $\mathrm{S}$ iteration processes.
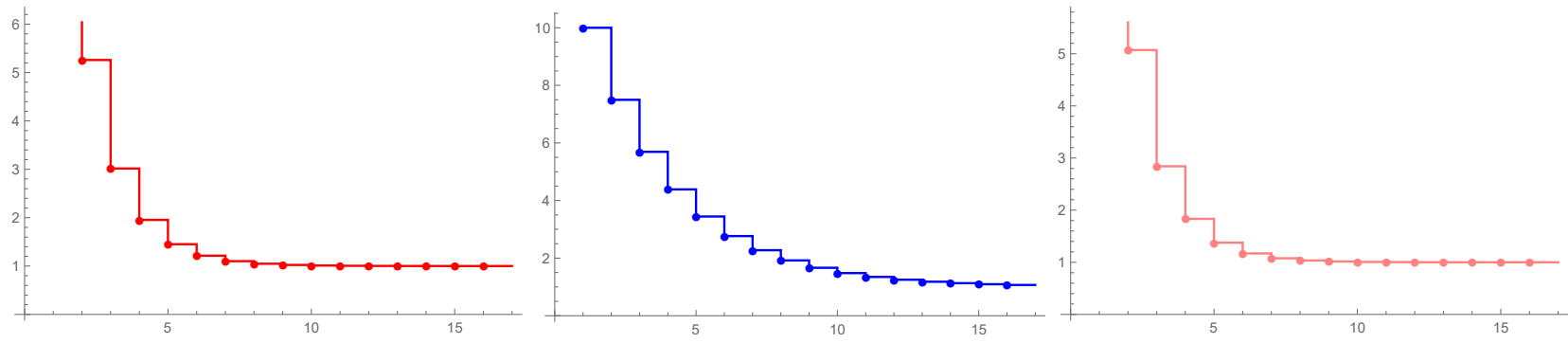

Figure 2: Graphical comparison of $\mathcal{V}_{n}$ for the function $\frac{1+4 x}{5}$ with $\alpha_{n}=0.75$ and $\beta_{n}=0.65$, and initial value $x_{0}=10$ (K*, $S$, and $\nu_{\mathrm{n}}$ iteration process). 


\section{An application}

Let a Banach space $\left(\mathcal{B}([a, b]),\|.\|_{\infty}\right)$ which is space of all continuous real valued functions on a closed interval $[a, b]$ a with endowed Chebyshev norm

$$
\|x-y\|_{\infty}=\max _{t \in[a, b]}|x(t)-y(t)| .
$$

In this section, solution of a particular delay differential equation has a solution generated by $\mathcal{V}_{\mathrm{n}}$ iteration scheme.

$$
\begin{aligned}
x^{\prime}(t) & =f(t, x(t), x(t-\tau)), \quad t \in\left[t_{0}, b\right], \\
x(t) & =\psi(t), \quad t \in\left[t_{0}-\tau, t_{0}\right] .
\end{aligned}
$$

We opine that the following conditions are performed

1. $\mathrm{t}_{0}, \mathrm{~b} \in \mathbb{R}, \tau>0$;

2. $f \in D\left(\left[t_{0}, b\right] \times \mathbb{R}^{2}, \mathbb{R}\right)$;

3. $\psi \in \mathrm{D}\left(\left[\mathrm{t}_{0}-\tau, \mathrm{b}\right], \mathbb{R}\right)$;

4. if $2 L_{f}\left(b-t_{0}\right)<1$, there exist $L_{f}>0$ such that

$$
\left|f\left(t, u_{1}, u_{2}\right)-f\left(t, v_{1}, v_{2}\right)\right| \leqslant L_{f} \sum_{n=0}^{2}\left|u_{i}-v_{i}\right|,
$$

$$
\forall u_{i}, v_{i} \in \mathbb{R}, i=1,2, t \in\left[\mathrm{t}_{0}, \mathrm{~b}\right]
$$

By a solution of the problem (5.1)-(5.2) we understand function $x \in D\left(\left[t_{0}-\tau, b\right], \mathbb{R}\right) \cap C^{1}\left(\left[t_{0}, b\right], \mathbb{R}\right)$. The problem (5.1)-(5.2) can be reformulated in the following form of integral

$$
x(t)=\psi(t), \quad t \in\left[t_{0}-\tau, t_{0}\right], \quad x(t)=\psi\left(t_{0}\right)+\int_{t_{0}}^{t} f(s, x(s), x(s-\tau)) d s, \quad t \in\left[t_{0}, b\right] .
$$

Theorem 5.1. Suppose that conditions 1-4 are satisfied. Then the problem (5.1)-(5.4) has a unique solution in $\mathrm{C}\left(\left[\mathrm{t}_{0}-\tau, \mathrm{b}\right], \mathbb{R}\right) \cap \mathrm{C}^{1}\left(\left[\mathrm{t}_{0}, \mathrm{~b}\right], \mathbb{R}\right)$.

Proof. Let $\left\{y_{n}\right\}_{\mathfrak{n}=0}^{\infty}$ be an iterative sequence generative by $\mathcal{V}_{\mathfrak{n}}$ iteration method (1.4) for the operator

$$
\wp x(t)=\psi(t), \quad t \in\left[t_{0}-\tau, t_{0}\right],
$$

or

$$
\wp x(t)=\psi\left(t_{0}\right)+\int_{t_{0}}^{t} f(s, x(s), x(s-\tau)) d s, \quad t \in\left[t_{0}, b\right] .
$$

Let $x_{\omega}$ denote the fixed point of $\wp$. We will show that $y_{n} \rightarrow x_{\omega}$ as $n \rightarrow \infty$. For $t \in\left[t_{0}-\tau, t_{0}\right]$, it is easy to see that $y_{n} \rightarrow x_{\omega}$ as $n \rightarrow \infty$. For $t \in\left[t_{0}, b\right]$ we obtain

$$
\begin{aligned}
\left\|x_{n}-x_{\omega}\right\|_{\infty} & =\left\|\wp\left(\left(1-\sigma_{n}^{0}\right) y_{n}+\sigma_{n}^{0} \wp y_{n}\right)-x_{\omega}\right\|_{\infty} \\
& =\left\|\omega\left(\left(1-\sigma_{n}^{0}\right) y_{n}+\sigma_{n}^{0} \wp y_{n}\right)-\wp x_{\omega}\right\| \\
& \leqslant\left\|\left(1-\sigma_{n}^{0}\right) y_{n}+\sigma_{n}^{0} \wp y_{n}-x_{\omega}\right\| \\
& \leqslant\left(1-\sigma_{n}^{0}\right)\left\|y_{n}-x_{\omega}\right\|_{\infty}+\sigma_{n}^{0} \max _{t \in\left[t_{0}-\tau, b\right]}\left|\wp y_{n}-\wp x_{\omega}\right|
\end{aligned}
$$

and hence,

$$
\left\|x_{n}-x_{\omega}\right\|_{\infty}=\left(1-\sigma_{n}^{0}\right)\left\|y_{n}-x_{\omega}\right\|_{\infty}+\sigma_{n}^{0} \max _{t \in\left[t_{0}-\tau, b\right]} \mid \psi\left(t_{0}\right)+\int_{t_{0}}^{t} f(s, x(s), x(s-\tau)) d s
$$




$$
\begin{aligned}
& -\psi\left(t_{0}\right)-\int_{t_{0}}^{t} f\left(s, x_{\omega}(s), x_{\omega}(s-\tau)\right) d s\left\|x_{n}-x_{\omega}\right\|_{\infty} \\
= & \left(1-\sigma_{n}^{0}\right)\left\|y_{n}-x_{\omega}\right\|_{\infty}+\sigma_{n}^{0} \max _{t \in\left[t_{0}-\tau, b\right]} \mid \int_{t_{0}}^{t} f(s, x(s), x(s-\tau)) d s \\
& -\int_{t_{0}}^{t} f\left(s, x_{\omega}(s), x_{\omega}(s-\tau)\right) d s \mid \\
= & \left(1-\sigma_{n}^{0}\right)\left\|y_{n}-x_{\omega}\right\|_{\infty}+\sigma_{n}^{0} \max _{t \in\left[t_{0}-\tau, b\right]} \int_{t_{0}}^{t} \mid f(s, x(s), x(s-\tau))
\end{aligned}
$$

which gives

$$
\begin{aligned}
-f & \left(s, x_{\omega}(s), x_{\omega}(s-\tau)\right) d s \mid \\
& =\left(1-\sigma_{n}^{0}\right)\left\|y_{n}-x_{\omega}\right\|_{\infty}+\sigma_{n}^{0} \max _{t \in\left[t_{0}-\tau, b\right]} \int_{t_{0}}^{t} L_{f}\left(\left|y_{n}(s)-x_{\delta}(s)\right|+\left|y_{n}(s-\tau)-x_{\omega}(s-\tau)\right|\right) d s \\
& =\left(1-\sigma_{n}^{0}\right)\left\|y_{n}-x_{\omega}\right\|_{\infty}+\sigma_{n}^{0} \max _{t \in\left[t_{0}-\tau, b\right]} \int_{t_{0}}^{t} L_{f}\left(\left|y_{n}(s)-x_{\delta}(s)\right|+\left|y_{n}(s-\tau)-x_{\omega}(s-\tau)\right|\right) d s \\
& =\left(1-\sigma_{n}^{0}\right)\left\|y_{n}-x_{\omega}\right\|_{\infty}+\sigma_{n}^{0} L_{f}\left(\max _{t \in\left[t_{0}-\tau, b\right]}\left|y_{n}(s)-x_{\delta}(s)\right|+\max _{t \in\left[t_{0}-\tau, b\right]}\left|y_{n}(s-\tau)-x_{\omega}(s-\tau)\right|\right) \int_{t_{0}}^{t} d s \\
& =\left(1-\sigma_{n}^{0}\right)\left\|y_{n}-x_{\omega}\right\|_{\infty}+2 \sigma_{n}^{0} L_{f}\left(b-t_{0}\right)\left\|y_{n}-x_{\omega}\right\|,
\end{aligned}
$$

and

$$
\left\|x_{n}-x_{\omega}\right\|_{\infty}=\left[1-\sigma_{n}^{0}\left(1-2 L_{f}\left(b-t_{0}\right)\right)\right]\left\|y_{n}-x_{\omega}\right\|,
$$

and hence,

$$
\left\|y_{n}-x_{\omega}\right\|_{\infty}=\left[\sigma_{n}^{1}-\sigma_{n}^{1}\left(1-2 L_{f}\left(b-t_{0}\right)\right)\right]\left\|z_{n}-x_{\omega}\right\|
$$

similarly, we have

$$
\begin{aligned}
\left\|z_{n}-x_{\omega}\right\|_{\infty} & =\left\|\wp x_{n}-\wp x_{\omega}\right\|_{\infty} \\
& \left.=\max _{t \in\left[t_{0}-\tau, b\right]} \mid \int_{t_{0}}^{t} f\left(s, x_{n}(s), x_{n}(s-\tau)\right)-f\left(s, x_{\omega}(s), x_{\omega}(s-\tau)\right)\right] d s \mid \\
& \leqslant \max _{t \in\left[t_{0}-\tau, b\right]} \int_{t_{0}}^{t}\left|f\left(s, x_{n}(s), x_{n}(s-\tau)\right)-f\left(s, x_{\omega}(s), x_{\omega}(s-\tau)\right)\right| d s \\
& =\max _{t \in\left[t_{0}-\tau, b\right]} \int_{t_{0}}^{t} L_{f}\left(\left|x_{n}(s)-x_{\omega}(s)\right|+\left|x_{n}(s-\tau)-x_{\omega}(s-\tau)\right|\right) d s, \\
\left\|z_{n}-x_{\omega}\right\|_{\infty} & \leqslant 2 L_{f}\left(b-t_{0}\right)\left\|x_{n}-x_{\omega}\right\|_{\infty},
\end{aligned}
$$

and hence, we have

$$
\left\|x_{n}-x_{\omega}\right\|_{\infty}=\left[1-\sigma_{n}^{0}\left(1-2 L_{f}\left(b-t_{0}\right)\right)\right]\left[\sigma_{n}^{0}-\sigma_{n}^{0}\left(1-2 L_{f}\left(b-t_{0}\right)\right)\right]\left[2 L_{f}\left(b-t_{0}\right)\left\|x_{n}-x_{\omega}\right\|_{\infty}\right],
$$

using the equations (5.5), (5.6), and (5.7),

$$
\left\|x_{n+1}-x_{\omega}\right\|_{\infty} \leqslant 8 L_{f}^{3}\left(b-t_{0}\right)^{2}\left[1-\sigma_{n}^{0}\left(1-2 L_{f}\left(b-t_{0}\right)\right)\right]\left\|x_{n}-x_{\omega}\right\| .
$$


Proceeding in the same manner, we have

$$
\left\|x_{n}-x_{\omega}\right\|_{\infty} \leqslant 8 L_{f}^{3}\left(b-t_{0}\right)^{2}\left[1-\sigma_{n-1}^{0}\left(1-2 L_{f}\left(b-t_{0}\right)\right)\right]\left\|x_{n-1}-x_{\omega}\right\|
$$

and

$$
\left\|x_{n-1}-x_{\omega}\right\|_{\infty} \leqslant 8 L_{f}^{3}\left(b-t_{0}\right)^{2}\left[1-\sigma_{n-2}^{0}\left(1-2 L_{f}\left(b-t_{0}\right)\right)\right]\left\|x_{n-2}-x_{\omega}\right\|
$$

and hence we have

$$
\left\|x_{n+1}-x_{\omega}\right\|_{\infty} \leqslant \prod_{k=0}^{n}\left[1-\sigma_{k}^{0}\left(1-2 L_{f}\left(b-t_{0}\right)\right)\right]\left\|x_{0}-x_{\omega}\right\|_{\infty}
$$

where $\left[1-\sigma_{k}^{0}\left(1-2 L_{f}\left(b-t_{0}\right)\right) \in(0,1)\right.$ because $\sigma_{k}^{0} \in(0,1)$, for all natural numbers $n$. Also, since $(1-x) \leqslant$ $e^{-x}$ for all $x \in[0,1]$, from (5.9) we can easily conclude that

$$
\left\|y_{n+1}-x_{\omega}\right\|_{\infty} \leqslant \frac{\left\|y_{0}-x_{\omega}\right\|}{e^{\left(1-\left(2 L_{f}\left(b-t_{0}\right)\right)\right) \sum_{k=0}^{\infty} \sigma_{k}^{0}}}
$$

which leads us to $\lim _{n \rightarrow \infty}\left\|y_{n+1}-x_{\omega}\right\|_{\infty}=0$, when taking limits of both sides of equation (5.10).

\section{References}

[1] M. Abbas, T. Nazir, Some new faster iteration process applied to constrained minimization and feasibility problems, Mat. Vesnik, 66 (2014), 223-234. 1

[2] R. P. Agarwal, D. O'Regan, D. R. Sahu, Fixed point theory for Lipschitzian-type mappings with applications, Springer, New York, (2009). 2

[3] R. P. Agarwal, D. O'Regan, D. R. Sahu, Iterative construction of fixed points of nearly asymptotically nonexpansive mappings, J. Nonlinear Convex Anal., 8 (2007), 61-79. 1, 1

[4] F. E. Browder, Nonexpansive nonlinear operators in a Banach space, Proc. Nat. Acad. Sci. U.S.A., 54 (1965), 1041-1044. 1

[5] D. Göhde, Zum Prinzip der Kontraktiven Abbildung, Math. Nachr., 30 (1965), 251-258. 1

[6] S. Ishikawa, Fixed points by a new iteration method, Proc. Amer. Math. Soc., 44 (1974), 147-150. 1

[7] W. A. Kirk, A fixed point theorem for mappings which do not increase distance, Amer. Math. Monthly, 72 (1965), 10041006. 1

[8] W. A. Mann, Mean value methods in iteration, Proc. Amer. Math. Soc., 4 (1953), 506-510. 1

[9] L. N. Mishra, V. Dewangan, V. N. Mishra, S. Karateke, Best proximity points of admissible almost generalized weakly contractive mappings with rational expressions on b-metric spaces, J. Math. Comput. Sci., 22 (2020), 97-109. 1

[10] M. A. Noor, New approximation schemes for general variational inequalities, J. Math. Anal. Appl., 251 (2000), $217-229$. 1

[11] Z. Opial, Weak convergence of the sequence of successive approximations for nonexpansive mappings, Bull. Amer. Math. Soc., 73 (1967), 591-597. 2.2

[12] B. E. Rhoades, Some fixed point iteration procedures, Internat. J. Math. Math. Sci., 14 (1991), 1-16. 1

[13] J. Schu, Weak and strong convergence to fixed points of asymptotically nonexpansive mappings, Bull. Amer. Math. Soc., 43 (1991), 153-159. 2

[14] H. F. Senter, W. G. Dotson, Approximating fixed points of nonexpansive mappings, Proc. Amer. Math. Soc., 44 (1974), 375-380. 3.6

[15] A. Sharma, M. Imdad, Approximating fixed points of generalized nonexpansive mappings via faster iteration schemes, Adv. Fixed Point Theory, 4 (2014), 605-623.

[16] T. Suzuki, Fixed point theorems and convergence theorems for some generalized nonexpansive mappings, J. Math. Anal. Appl., 340 (2008), 1088-1095. 1, 2, 2.4

[17] W. Takahashi, Nonlinear Functional Analysis, Yokohoma Publishers, Yokohoma, (2000). 2

[18] B. S. Thakur, D. Thakur, M. Postolache, A new iterative algorithm for numerical reckoning fixed points of Suzuki's generalized nonexpansive mappings, Appl. Math. Comput., 275 (2016), 147 pages. 1, 1

[19] K. Ullah, M. Arshad, New three-step iteration process and fixed point approximation in Banach spaces, J. Linear Topol. Algebra, 7 (2018), 87-100. 1 